\title{
Gaussian approximation of the bunch lengthening in electron storage rings with a typical wake function
}

\author{
S. Petracca, ${ }^{1}$ Th. Demma, ${ }^{1}$ and K. Hirata ${ }^{2}$ \\ ${ }^{1}$ University of Sannio, Benevento, Italy and INFN, Salerno, Italy \\ ${ }^{2}$ Sokendai, The Graduate University for Advanced Studies, Hayama, Japan
}

(Received 3 April 2005; published 6 July 2005)

\begin{abstract}
The validity of the Gaussian approximation of the distribution function in the study of the equilibrium bunch length in electron storage rings is considered with specific reference to the inductive wake. The Gaussian approximation can be used to describe not only localized wakes, but also uniformly distributed wakes, for which the longitudinal profiles at equilibrium are ruled by the potential-well-distortion (Haissinski) equation. Comparison of Gaussian approximation based results with those obtained from Haissinski equation shows good qualitative agreement in their common range of validity, while shedding light on the nature of the unstable regimes.
\end{abstract}

DOI: $10.1103 /$ PhysRevSTAB.8.074401

PACS numbers: 29.20.-c, 29.27.Bd

\section{INTRODUCTION}

The wake force describes space-charge and image interactions which affect the particles distribution in a bunched beam [1]. At low current, the longitudinal equilibrium distributions can be obtained in analytic form by solving the potential-well-distortion (PWD) equation named after Haissinski [2]. The Haissinski equation is a complicated nonlinear integral equation. A linear stability analysis around the static solutions gives the threshold for turbulent bunch lengthening. Obviously, this tells nothing about the nature of the unstable regimes.

On the other hand it is more realistic, especially for large machines, to consider the many sources of wake forces along the ring as localized, as usually assumed in multiparticle-tracking (MPT) codes. One of the authors introduced an analytic model for describing localized (timedependent) wake forces [3] . The main simplifying assumption is that if the initial distribution function in synchrotron phase space is Gaussian, it can still be approximated by a Gaussian after the action of the wake force, represented by a "kick." The distribution function can be accordingly parametrized by its first and second order moments only, describing the beam envelope in synchrotron phase space. The evolution of these moments from turn to turn is obtained by applying a (nonlinear) mapping at each turn. Remarkably, even in cases when the actual longitudinal distribution is not unimodal, the Gaussian approximation proved to be not only capable of capturing the physics of the problem (e.g., multistable regimes, bifurcations, etc.) but also of predicting accurately the relevant critical parameter values, by comparison with MPT based results [4].

The localized-wake assumption can be suitably extended so as to model the uniformly distributed wake case, to which Haissinski equation applies. To do so one introduces $N_{s}$ localized (equally spaced) kicks with (equal) strengths $\propto N_{s}^{-1}$, and eventually lets $N_{s} \rightarrow \infty$.
This allows us to make a direct comparison between the Haissinski and Gaussian approximation approaches - with the added value that this latter provides insight into the nature of the unstable regimes.

For a constant wake function (capacitive beam coupling impedance), a solution of the Haissinski equation always exists [5]. On the other hand, the Gaussian approximation approach reveals the possible coexistence of multistable states in a cusp-catastrophe scenario [6], for certain values of the relevant machine parameters, in close agreement with MPT based simulations. Similarly, for a $\delta$ wake function (resistive beam coupling impedance) a solution of the Haissinski equation always exists [7], and the Gaussian approximation (map) approach discloses multistable states in some regions of parameter space [8].

In this paper the Gaussian approximation (map) and Haissinski (equation) approach will be compared and contrasted for the specific case of a $\delta^{\prime}$ wake function (inductive beam coupling impedance). For a $\delta^{\prime}$ wake function a solution of Haissinski equation is known to exist only below a certain current-threshold [9]. On the other hand, it has been recently shown [10] that after a suitable physically motivated regularization of the singularity, a solution of the Haissinski equation with $\delta^{\prime}$ wake function always exists too.

The paper is accordingly laid out as follows. In Sec. II we introduce the moment mapping, specialized to the inductive $\left(\delta^{\prime}\right)$ wake case. In Sec. III we discuss Haissinski equation for the same wake. In Sec. IV we compare the two approaches. In Sec. V we introduce the regularized inductive wake function. Conclusions follow under Sec. VI.

\section{THE GAUSSIAN APPROXIMATION}

The longitudinal beam dynamics in electron storage rings can be described by single-particle stochastic (Langevin) equations of motion [3] in the canonical vari- 
ables:

$$
\begin{aligned}
& x_{1}=\frac{\text { longitudinal displacement }}{\text { natural bunch length }}, \\
& x_{2}=\frac{\text { relative energy spread }}{\text { natural energy spread }} .
\end{aligned}
$$

Integrating the Langevin equations over one turn, one obtains the following stochastic mapping:

$$
\left(\begin{array}{l}
x_{1} \\
x_{2}
\end{array}\right)^{\prime}=U\left(\Lambda x_{2}+\hat{r} \sqrt{1-\Lambda_{1}}-\phi\left(x_{1}\right)\right),
$$

where $\vec{X}^{\prime}=\left(x_{1}^{\prime}, x_{2}^{\prime}\right)$ is $\vec{X}=\left(x_{1}, x_{2}\right)$ after one turn. Here $U$ is the rotation matrix:

$$
U=\left(\begin{array}{cc}
\cos \mu & \sin \mu \\
-\sin \mu & \cos \mu
\end{array}\right)
$$

$\mu=2 \pi \nu_{s}, \quad \nu_{s}$ being the synchrotron tune, $\Lambda=$ $\exp (-2 / T), T$ the synchrotron damping time measured in units of the revolution period, and $\hat{r}$ a Gaussian random variable with $\langle\hat{r}\rangle=0$ and $\left\langle\hat{r}^{2}\right\rangle=1$. The wake force (integrated over one turn) is represented by

$$
\phi\left(x_{1}\right)=\int_{0}^{\infty} \rho(x-u) \tilde{W}(u) d u,
$$

where $\rho(x)$ is the charge density normalized to one, and

$$
\tilde{W}(x)=\frac{q^{2} N}{\sigma_{E}} W(x),
$$

$W(x)$ being the (monopole) longitudinal wake function [volt/coulomb], $N$ the total number of particles in the bunch and $\sigma_{E}$ the nominal (absolute) energy spread. Note that in Eq. (2.2) synchrotron oscillations have been linearized, and radiation is localized at one point of the ring [11]. It is also understood that $W_{L}$ is such that multiturn effect can be neglected. The above stochastic mapping is equivalent to an infinite hierarchy of deterministic maps in the moments of the distribution function $\psi(\vec{x})$, i.e.,

$$
\bar{x}_{i}=\left\langle x_{i}\right\rangle, \quad \sigma_{i j}=\left\langle\left(x_{i}-\bar{x}_{j}\right)\left(x_{j}-\bar{x}_{j}\right)\right\rangle, \quad \text { etc. },
$$

where $\langle\cdot\rangle$ indicates average over all particles. We now make the main assumption that the distribution function in phase space can be approximated by a Gaussian, even in the presence of a wake force,

$$
\psi\left(x_{1}, x_{2}\right)=\frac{\exp \left[-\frac{1}{2} \sum_{i, j}^{2} \sigma_{i j}^{-1}\left(x_{i}-\bar{x}_{i}\right)\left(x_{j}-\bar{x}_{j}\right)\right]}{2 \pi \sqrt{\operatorname{det}\left[\sigma_{i j}\right]}},
$$

and use (2.7) to compute the averages in (2.6). See Secs. 5.3 and 5.4 in [3] for a discussion of the physical implications and limitations of the Gaussian approximation.

For the special case of the $\delta^{\prime}$ wake function, of interest here,

$$
\tilde{W}(x)=-b \delta^{\prime}(x)
$$

(purely inductive beam coupling impedance), we can split the maps for the second order moments into three parts, representing the effect of radiation, wake force, and synchrotron oscillation, respectively, as follows:

$$
\begin{aligned}
& \sigma_{11}^{\prime}=\sigma_{11}, \quad \sigma_{12}^{\prime}=\Lambda \sigma_{12}, \\
& \sigma_{22}^{\prime}=\Lambda^{2} \sigma_{22}+\left(1-\Lambda^{2}\right) \quad \text { (radiation); }
\end{aligned}
$$

$$
\begin{aligned}
& \sigma_{11}^{\prime}=\sigma_{11}, \quad \sigma_{12}^{\prime}=\sigma_{12}+\frac{b}{4 \sqrt{\pi \sigma_{11}}} \\
& \sigma_{22}^{\prime}=\sigma_{22}+\frac{b \sigma_{12}}{2 \sigma_{11} \sqrt{\pi \sigma_{11}}}+\frac{b^{2}}{6 \sigma_{11}^{2} \pi \sqrt{3}} \quad \text { (wake force) }
\end{aligned}
$$

$$
\sigma_{i j}^{\prime}=\sum_{h, k=1}^{2} U_{i h} \sigma_{h k} U_{k j}^{t}, \quad \text { (synchrotron oscillation), }
$$

where $U$ is the rotation matrix (2.3) and $U^{t}$ its transpose. The stability and nature of the solutions of the resulting product map depends on the values of the synchrotron tune $\nu_{s}$, the damping time (measured in number of turns) $T$, and the strength of the wake force, represented by $b$.

We explored systematically a wide range of (meaningful) parameters values [12], and found stable solution of period one and higher, in agreement with previous findings in [8], but also finely cascaded bifurcations and chaos for some parameters values. As an illustration, in Fig. 1 the pertinent regions of a $(b, T)$ parameter space subset are shown for $\nu_{s}=0.085$. The existence and size of the chaotic region turns out to be weakly dependent on the value of $\nu_{s}$. As $b$ decreases through negative values, the map exhibits a bifurcation-cascade route to chaos. This is illus-

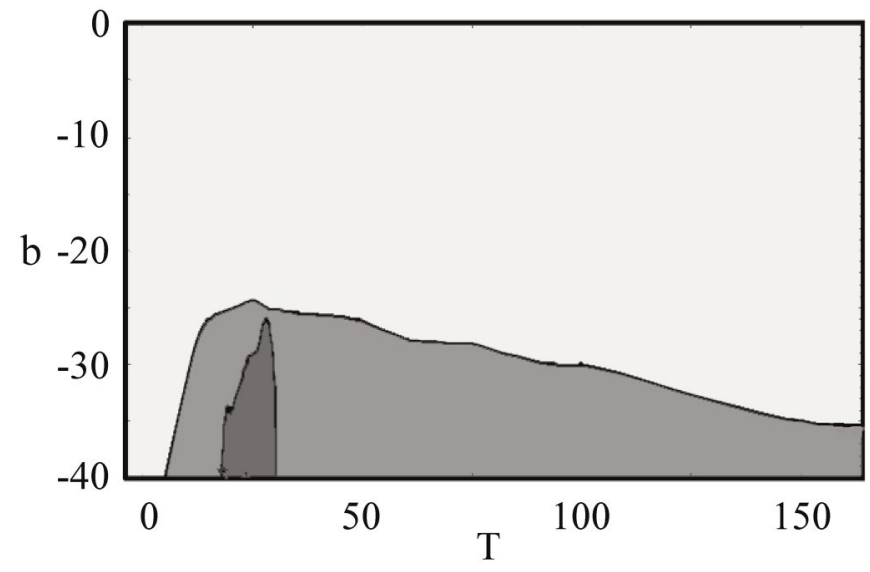

FIG. 1. Gaussian approximation with $\delta^{\prime}$ wake. Nature of the solutions in parameter subspace $\{b, T\}$ with $\nu_{s}=0.085$. Light gray: period-1; dark-gray: period-2 or higher; medium-gray: chaotic. 



FIG. 2. Gaussian approximation with $\delta^{\prime}$ wake. $\sigma_{i j}$ versus $b<0$ for $T=200, \nu_{s}=0.085$, and $N_{S}=1$.

trated in Fig. 2, where the second order moments $\sigma_{i j}$ are displayed as functions of $b$, for $T=200$ and $\nu_{s}=0.085$.

\section{THE HAISSINSKI EQUATION}

Let us introduce the synchrotron variables

$$
\epsilon=\left(E-E_{s}\right) / E_{s}, \quad \xi=\omega_{s} \tau / \alpha \sigma_{\epsilon},
$$

where $E$ is the energy of the single-particle, the suffix $s$ indicates the synchronous particle with the nominal energy $E_{s}, \omega_{s}$ is the synchrotron angular frequency, $\tau=$ $\left(z-z_{s}\right) / \beta c$ is the time displacement with respect to the synchronous particle, $\beta$ is the relativistic factor, $c$ is the light velocity in vacuum, $\alpha$ is the momentum compaction factor, and $\sigma_{\epsilon}$ is the relative energy spread. The static solution of the Fokker-Planck equation for the phase space particle distribution can be written:

$$
\psi(\epsilon, \xi)=\rho(\xi) \exp \left\{-\frac{\epsilon^{2}}{2 \sigma_{\epsilon}^{2}}\right\}
$$

The (dimensionless) longitudinal density $\rho(\xi)$ in Eq. (3.2) is a solution of the Haissinski equation [13],

$$
\rho(\xi)=A \exp \left\{-\frac{\xi^{2}}{2}+\int_{\xi}^{+\infty} V\left(\xi^{\prime}\right) d \xi^{\prime}\right\}
$$

where $A$ is a normalization constant such that:

$$
\int_{-\infty}^{+\infty} \rho(\xi) d \xi=1
$$

and

$$
V(\xi)=\int_{\xi}^{+\infty} \rho\left(\xi^{\prime}\right) w\left(\xi^{\prime}-\xi\right) d \xi^{\prime}
$$

with

$$
w(\xi)=\frac{q^{2} N}{2 \pi \nu_{s} \sigma_{E}} W(\xi) .
$$

$W(\xi)$ being the (monopole) longitudinal wake function [volt/Coulomb]. Letting

$$
w(\xi)=2 S \delta^{\prime}(\xi)
$$

Eq. (3.3) becomes

$$
\frac{\rho^{\prime}(\xi)}{\rho(\xi)}=-\xi+S \rho^{\prime}
$$

whose solution is

$$
\log \rho(\xi)-S \rho(\xi)=-\frac{\xi^{2}}{2}+\log A
$$

where $A$ is an arbitrary constant whose value is chosen so as to cope with (3.4). The solution of Eq. (3.9) can be written explicitly in terms of the Lambert $W$ function [14], defined by the series expansion:

$$
W_{L}(z)=\sum_{n=0}^{\infty} \frac{(-n)^{n-1}}{n !} z^{n}, \quad|z|<\exp (-1)
$$

and is

$$
\rho(\xi)=-\frac{W_{L}\left[-A S \exp \left(-\xi^{2} / 2\right)\right]}{S} .
$$

It is well known $[9,10]$ that for $S \leq 0$ a solution of (3.3) satisfying Eq. (3.4) always exists. On the other hand, if

$$
S>S^{*} \approx 1.55061
$$

no value of $A$ can satisfy the normalization condition (3.4), and no stable solution of Haissinski equation does accordingly exists [15].

\section{COMPARISON}

The localized-wake assumption on which the Gaussian approximation is based can be extended to model the uniformly distributed wake case to which Haissinski equation applies, so as to make a comparison possible. This is obtained by making the following substitutions in the map:

$$
\nu_{s} \longrightarrow \nu_{s} / N_{s}, \quad T \longrightarrow T N_{s}, \quad b \longrightarrow b / N_{s},
$$

where the superperiodicity index $N_{s}$ is eventually let grow 

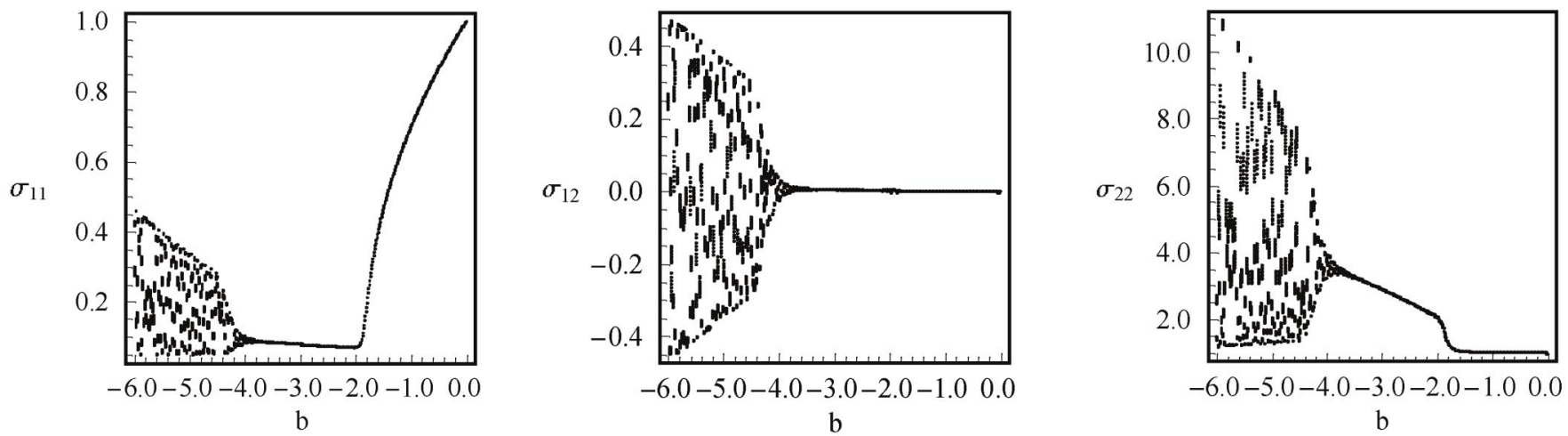

FIG. 3. Gaussian approximation with $\delta^{\prime}$ wake. $\sigma_{i j}$ versus $b<0$ for $T=200, \nu_{s}=0.085$ and $N_{s}=150$.

to infinity, thus distributing the wake force among an increasing number of (equally spaced) kicks along the ring.

It is interesting to compare Fig. 3, where $N_{s}=150$, to Fig. 2, where $N_{s}=1$. A cascaded bifurcation route to chaos is again observed, except that here the instability sets in at a much smaller value of $-b$. For sufficiently large $N_{s}$, the threshold $b^{*}$ corresponds precisely to the threshold $S^{*}$ above which the solution of the Haissinski equation does not exist. Indeed, $S$ and $b$ are more or less obviously related by $b=\chi S$, where the constant $\chi=-4 \pi \nu_{s}$. This is most readily seen by assuming a $\delta^{\prime}$ longitudinal wake with strength $W_{0}$ first in (3.6), so as to obtain from (3.7)

$$
S=W_{0} \frac{q^{2} N}{4 \pi \nu_{s} \sigma_{E}}
$$

and then in (2.5), so as to obtain from (2.8)

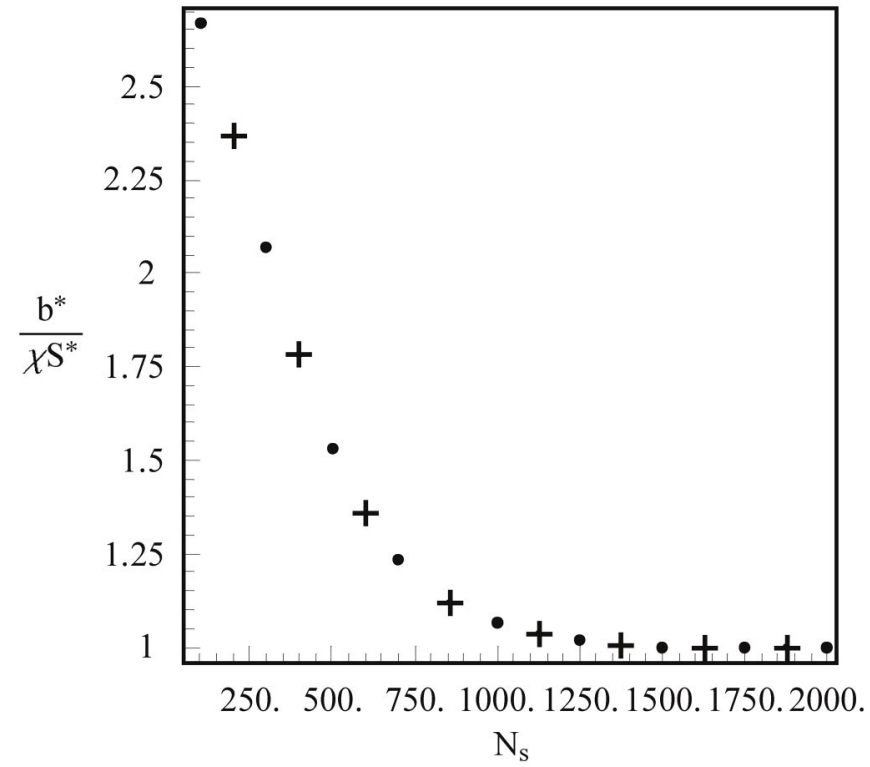

FIG. 4. Gaussian approximation with $\delta^{\prime}$ wake. The ratio $b^{*} / \chi S^{*}$ versus $N_{s}$ for $T=200, \nu_{s}=0.085$ (dots) and $\nu_{s}=$ 0.2 (crosses).

$$
b=-\frac{q^{2} N}{\sigma_{E}} W_{0},
$$

and finally eliminating $W_{0}$ between (4.2) and (4.3). In Fig. 4 the ratio $b^{*} / \chi S^{*}$ is plotted as a function of the superperiodicity $N_{s}$, for two different values of $\nu_{s}$. As $N_{s}$ is increased, the ratio gets closer and closer to unity.

On the other hand, for $b>0(S<0)$, a solution of Haissinski equation always exists, and agrees fairly well with the solutions obtained from the Gaussian approximation for $N_{s} \gg 1$. This is illustrated in Fig. 5 where $\sigma_{11}$ is plotted vs $b>0$, for different values of $N_{s}$. As $N_{s}$ increases the curves become more and more closely spaced.

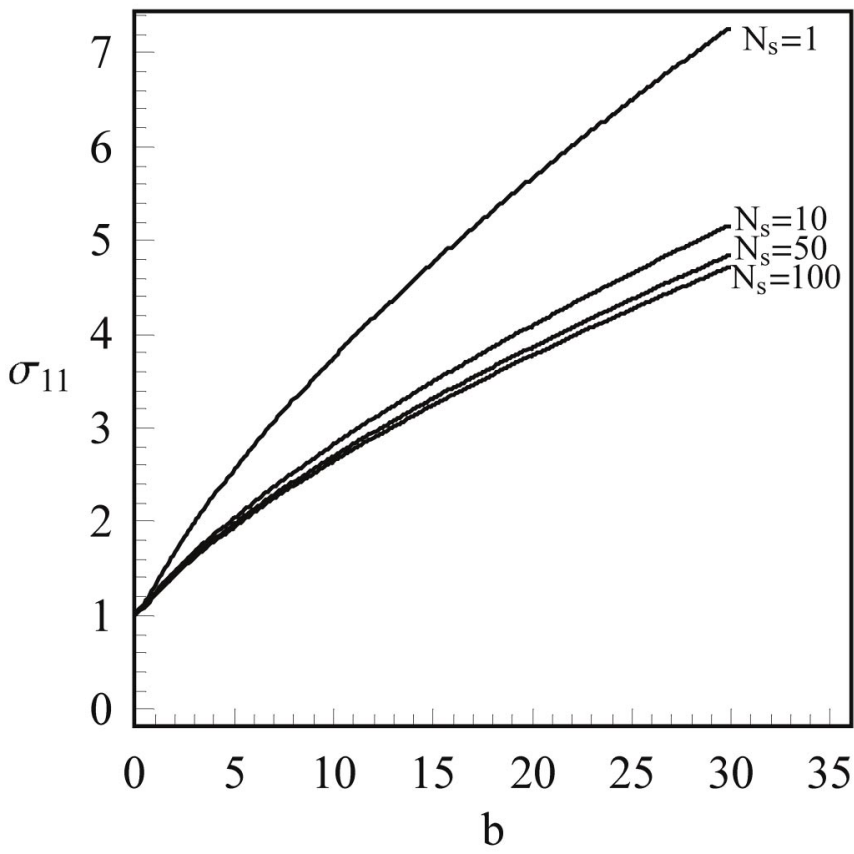

FIG. 5. Gaussian approximation with $\delta^{\prime}$ wake. $\sigma_{11}$ versus $b>$ 0 for various $N_{s}$, with $T=30$ and $\nu_{s}=0.085$. The curve derived from Haissinski equation is indistinguishable from those with $N_{s} \geq 100$. 
Eventually $\sigma_{11}$ converges to the bunch length (rms spread) of the Haissinski equation solution [16].

\section{REGULARIZED WAKE}

The wake function $\delta^{\prime}$ was regularized in [10] on the basis of a simple but sound physical argument by replacing Eq. (2.8) with

$$
\tilde{W}_{\mathrm{reg}}(x)=-b \lim _{a \rightarrow 0+} \frac{\delta(x)-\delta(x-a)}{a},
$$

where $a$ is a positive parameter, so as to satisfy the causality condition whereby each particle is influenced only by those which precede it, but not by those which follow. It was shown in [10] that using the above regularized inductive wake, the Haissinski equation has a solution for any value of $S$.

The mapping for the wake force obtained using (5.1) in place of (2.8) reads

$$
\begin{aligned}
\sigma_{11}^{\prime}= & \sigma_{11}, \quad \sigma_{12}^{\prime}=\sigma_{12}+\frac{b \exp \left(-a^{2} / 4 \sigma_{11}\right)}{4 \sqrt{\pi \sigma_{11}}}, \\
\sigma_{22}^{\prime}= & \sigma_{22}+\frac{b \sigma_{12} \exp \left(-a^{2} / 4 \sigma_{11}\right)}{2 \sqrt{\pi \sigma_{11}} \sigma_{11}} \\
& -\frac{b^{2}\left[1-\exp \left(-a^{2} / 4 \sigma_{11}\right)\right]^{2}}{4 a^{2} \sigma_{11} \pi} \\
& +\frac{b^{2}\left[1+\exp \left(-a^{2} / \sigma_{11}\right)-2 \exp \left(-a^{2} / 3 \sigma_{11}\right)\right]}{2 \sqrt{3} a^{2} \sigma_{11} \pi} .
\end{aligned}
$$

We explored a wide range of parameter values and found that using the physically regularized inductive wake (5.1) the Gaussian approach also yields regular (nonchaotic) solutions (both for $N_{s}=1$ and $N_{s} \gg 1$ ) for any value of $b$. As an illustration, in Fig. 6 we display $\sigma_{11}$ vs $b$ for $T=$ $200, \nu_{s}=0.085$, and $N_{s}=150$, for several values of $a$. It is seen that the physical regularization of the $\delta^{\prime}$ wake suppresses the instabilities.

\section{CONCLUSIONS}

Previous comparisons between the Gaussian approximation and the Haissinski equation for the special relevant cases of constant and $\delta$ wake functions, corresponding to capacitive and resistive beam coupling impedances, respectively, show that the two approaches agree quite well in their common range of validity, under the superperiodicity approximation for a continuously distributed wake.

Here we investigated in some detail the Gaussian approximation for a $\delta^{\prime}$ wake function (purely inductive beam coupling impedance). We found that for $b>0$ in (2.8) the Gaussian approximation yields stable periodic solutions for any $N_{s} \geq 1$ [8]. For $b<0$ the Gaussian approximation yields stable solutions, for any $N_{s} \geq 1$, for $b$ above a critical value $b^{*}$, which depends on $N_{s}$. Below this critical

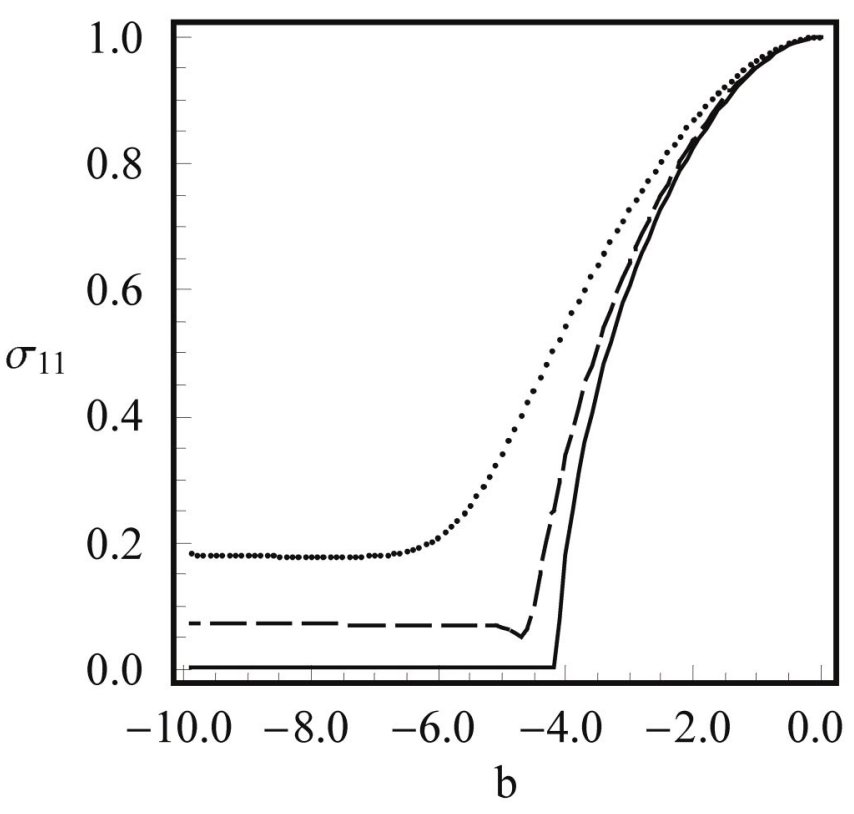

FIG. 6. Gaussian approximation with physically regularized $\delta^{\prime}$ wake, Eq. (5.1). $\sigma_{11}$ vs $b<0$ for $a=0.1$ (dotted line), $a=0.05$ (dashed line), $a=0.01$ (solid line), with $T=200, \nu_{s}=0.085$, and $N_{s}=150$.

value, a cascaded bifurcation route to chaos shows up. By suitably increasing $N_{s}$, the critical value $b^{*}$ converges rather rapidly to a value which corresponds exactly to the critical value of $S$ beyond which the Haissinski equation has no solution. For a physically regularized (causal) $\delta^{\prime}$ wake function, on the other hand, for which the existence of solutions of Haissinski equation (stable longitudinal profiles) is guaranteed for any current, the Gaussian approximation also yields stable solutions, for any $N_{s} \geq 1$, throughout the parameter space.

It should be emphasized that while the Haissinski equation can merely yield the stable solutions, or predict their nonexistence, the Gaussian approximation provides significantly deeper physical insight, by revealing the nature of the instabilities (e.g., multistable regimes, period-doubling bifurcations, and chaos), as well as the parameter space regions where they occur-a very important issue in beam dynamics analysis. On the basis of available results, we suggest that the Gaussian approximation may provide a reliable analytic framework to gain insight into the beam dynamics, aside of massive numerical simulations based on MPT codes [17].

In this connection we note that a superposition $\alpha \delta^{\prime}(x)+$ $\beta \delta(x)+\gamma$ could be used to model a wide class of wake functions (including the broadband resonant case). Unfortunately, in view of the nonlinearity of the Gaussian approximation approach, no straightforward conclusions can be drawn from the above summarized results for this more general case. Further quantitative investigation is accordingly in order. 
[1] S. Heifets and S. Kheifets, Rev. Mod. Phys. 63, 631 (1991).

[2] K. Haissinski, Nuovo Cimento B 18, 72 (1973).

[3] K. Hirata, Part. Accel. 22, 57 (1987).

[4] K. Hirata, S. Petracca, and F. Ruggiero, CERN Report No. SL/90-96 AP, 1990.

[5] Y. Shobuda and K. Hirata, Phys. Rev. E 64, 067501 (2001).

[6] K. Hirata, S. Petracca, and F. Ruggiero, Phys. Rev. Lett., 66, 1693 (1991).

[7] A. G. Ruggiero, IEEE Trans. Nucl. Sci. 241205 (1977).

[8] K. Hirata and E. S. Kim, KEK Report No. 95-5, 1995.

[9] A. W. Chao, Physics of Collective Beam Instabilities in High Energy Accelerators (Wiley Interscience, New York, 1993).

[10] Y. Shobuda and K. Hirata, Phys. Rev. E 60, 2414 (1999); also Y. Shobuda and K. Hirata, Part. Accel. 62, 165 (1999).
[11] K. Hirata and F. Ruggiero, Part. Accel. 28, 137 (1990).

[12] S. Petracca and Th. Demma, in Proceedings of the 2003 Particle Accelerator Conference, edited by J. Chew, P. Lucas, and S. Webbers (IEEE Press, Piscataway, 2003), p. 2996.

[13] S. Petracca, Part. Accel. 42, 45 (1993).

[14] C. Thomas et al., Europhys. Lett. 60, 66 (2002).

[15] Equation (3.12) is most readily established by noting that $A=\exp (-1) / S$ at the border of the convergency circle of (3.10), and using the corresponding maximum of $A S=$ $\exp (-1)$ in (3.11) to enforce (3.4).

[16] Note that (as seen from Figs. 2 and $3 \sigma_{12} \approx 0$ for $b>0$, so that $\sigma_{11}$ coincides with the rms width of the longitudinal profile of the Gaussian approximation solution.

[17] MPT codes require very fine meshings (and a very large number of particles) to deal with $\delta$ and $\delta^{\prime}$ wakes, which eventually makes them inefficient. 